\title{
O NEUROLOGISTA E A LITERATURA MÉDICA: O PROCESSO DE EDUCAÇÃO INTERMINÃVEL
}

\author{
MARCO AURELLIO LANA-PEIXOTO*
}

\begin{abstract}
RESUMO - A grande profusão da literatura médica gerou a necessidade do desenvolvimento de técnicas de seleção e de leitura que permitem o acesso e a avaliação crítica dos trabalhos mais significativos em relação à atualização e ao aperfeiçoamento das nossas habilidades na prática diária. A prioridiade está na leitura de artigos originais, ou seja, daqueles que relatam novas observações ou resultados de investigações planejadas. Por sua vez, os artigos originais devem ser selecionados a partir da avaliação de sua aplicabilidade à nossa prática especifica e do rigor de sua metodologia. $O$ acesso aos artigos originais relevantes ocorre por seíeção dos periódicos que serão abordados. A leitura das principais revistas da especialidade, o manuseio das revistas gerais de maior circulação e com maior fator impacto, e a consulta regular aos Current Contents são os métodos mais eficazes de triar os artigos que serão então avaliados. A Ieitura de uma revista nacional da especialidade tem interesse para se acompanhar o desenvolvimento da neurologia no país e para o estudo da epidemiologia e das peculiaridades das doenças prevalentes em nosso meio. Os artigos de revisão da literatura são úteis apenas como abordagem geral de um tema, como fonte de referências de trabalhos originais ou quando contêm meta-análise dos artigos incluídos.
\end{abstract}

The neurologist and the medical literature: the endless education process.

SUMMARY - To keep up to date with new advances in medical care and in the understanding of the disease process which affects their specific patients neurologists need to develop strategies for continuing education. They must be learners throughout their careers and the learning must be self-directed, active and independent. The best way to reach this goal is to devote regular time to the medical literature. However as the great volume of journal literature precludes clinicians from reading all of it, special tactics of scanning, selecting and reading medical articles are necessary. Priority should be given to original reports, meaning firsthand accounts of planned investigations, as they go through expert critical review before publication. This safeguard improves their clarity and usually keeps flawed research away. Furthermore one should read only original articles that have direct bearing on his ows clinical practice and with scientific rigor making the results likely to be true. Employment of these rules may reduce the relevant literature to an affordable size. Extraction of these articles depend on the regular surveillance of high-yield, high-circulation general medical journals and the specialty and subspecialty journals which are relevant to one's practice. Review articles may be helpful either as an initial approach to a subject whose current knowledge is beyond one's usual domain or as a source of references of original articles. Meta-analysis or the integration and statistical combination of the results of previous research may be a further and a most important advantage of reviews.

* Professor Adjunto de Neurologia, Faculdade de Medicina da Universidade Federal de Minas Gerais (UFMG).

Dr. Marco Aurélio Lana Peixoto - Instituto Hilton Rocha - Av. Anel da Serra 1355 - 30210 Belo Horizonte MG - Brasil. 
It is astonishing with how little reading a doctor can practice Medicine but it is not astonishing how badly he may do it.

É inestimável o benefício que o progresso das ciências biomédicas traz para nossa prática diária, tanto em relação à compreensão dos fenômenos da doença quanto à qualidade da assistência que oferecemos a nossos pacientes. No entanto, o volume de informações é tão grande e publicado em um sem-número de diferentes periódicos que muitos médicos se sentem frequentemente impotentes e desorientados diante das estantes das bibliotecas. Mais de dois milhões de artigos são publicados anualmente nas revistas biomédicas 1 .

O primeiro sentimento que o médico pode ter ao deparar com os recém-chegados números das revistas médicas em uma biblioteca é o de espanto e de admiração, seguidos logo pela triste consciência de sua incapacidade de ler e acompanhar todo o desenvolvimento das diferentes áreas da medicina. O simples passar de olhos sobre os indices de alguns periódicos the mostrará, de imediato, quão desatualizados já se encontram seus conhecimentos até pela dificuldade de compreensão de alguns títulos dos artigos ali dispostos. As palavras já não são familiares, os novos termos soam agressivos, e aquelas doenças listadas comportadamente em seus livros-textos agora lhe parecem rebeladas, inexoravelmente soltas da estrutura de seu conhecimento, ameaçando sua própria auto-confiança.

É tão desagradável esta percepção que muitos tombam ao primeiro embate. A literatura médica passa ser vista como esotérica, distante da realidade terrena de nossa vida profissional. As visitas às bibliotecas tornam-se então raras, a maioria das vezes apenas para a busca de uma referência específica. E as soluções dos problemas da prática do dia-a-dia, nas enfermarias e consultórios, serão ainda procuradas nos livros-textos e monografias. O resultado de tal comportamento é a dissociação, cada vez maior, entre a qualidade da assistência efetivamente prestada aos doentes e aquela possível e devida, baseada em novos conceitos advindos de trabalhos realizados e já publicados. O lapso de tempo entre uma informação publicada em um periódico até seu aparecimento em livro-texto é de anos, já que entre a pena do autor e a edição de um livro transcorrem, em geral, mais de dois anos 9. Até que o livro chegue às nossas mãos é possível que os outros dois anos também já tenham passado! Isto sem contar que a latência para a publicação de um artigo original após o seu término representa cerca de $25 \%$ do tempo desde a idéia inicial da pesquisa até o aparecimento do artigo na revista 20.

A desatualização dos médicos é fato inegável e, em certo sentido, inevitável ante a velocidade das transformações do conhecimento. $O$ comparecimento a cursos e congressos é medida pela qual os médicos procuram reduzir estas distâncias científicas. No entanto, por dependerem de tempo e de recursos financeiros maiores, as idas a cursos e congressos, na maioria das vezes fora do país, não se tornam medida eficaz e prática. $\mathrm{O}$ relacionamento constante, regular e metódico com a literatura médica se coloca então como a maneira mais eficiente, mais exequível e menos dispendiosa pela qual podemos nos manter atualizados em nossa prática profissional.

O diálogo ativo que se trava com os autores e pesquisadores a partir do momento em que se seleciona um determinado trabalho para leitura, ao se identificar a relevância daquele artigo para a solução de problemas da prática, ao analisar criticamente a metodologia usada, verificar os resultados obtidos e acompanhar os autores através da discussão de seus dados em relação aos conceitos anteriormente estabelecidos e, dai então, comparar os achados às próprias observações e idéias é aventura intelectual capaz de proporcionar além de grande satisfação, mudanças de atitudes e comportamentos, aperfeiçoando as habilidades profissionais. A atividade de leitura dos artigos originais publicados nos periódicos médicos é, portanto, o processo de educação constante $\mathrm{e}$ interminável ao longo da vida daqueles que pretendem oferecer a si próprios melhor compreensão dos fenômenos da doença e a seus pacientes melhor orientação diagnóstica e terapêutica. 
O objetivo deste artigo é formular e rever alguns princípios gerais e métodos básicos pelos quais os neurologistas, em sua prática diária, possam se beneficiar, pelo relacionamento com a literatura médica. Aqui é abordado o que ler, ou seja, como selecionar os artigos originais relevantes à prática, como identificar aqueles que merecem ser lidos pelo adequado rigor científico de sua metodologia e cujos resultados possam, portanto, ser confiáveis; quais revistas médicas devem ser rastreadas à procura destes trabalhos. Por fim, são feitas algumas considerações a respeito dos artigos de revisão da literatura, quais as suas utilidades e como identificar aqueles cuja leitura possa trazer benefícios para a prática. Em artigo complementar serão discutidas algumas técnicas e sugestões para a leitura dos artigos científicos. Os princípios gerais e as regras básicas dos vários tipos de leitura - leituras de inspeção, analítica, crítica e comparativo-combinada - serão então considerados como método sistemático no relacionamento ativo com a literatura médica.

\section{$O$ que ler para se manter atualizado}

Todos nós podemos nos considerar atrasados em nossas leituras. Há sempre mais o que ler do que nosso tempo nos permite. Para ilustrar este ponto Warren 22 demonstra que um clínico para acompanhar as 10 mais importantes revistas de clínica médica deve ler 200 artigos e 70 editoriais por mês. Isto para não dizer que para ler tudo de interesse biomédico que é publicado ele teria que ler mais que 5500 artigos por dia 9. Estes dados mostram a necessidade de selecionar os trabalhos por métodos objetivos e rápidos, de modo que nosso tempo seja dispendido com os trabalhos que: (1) possam ser relevantes à nossa prática; e (2) que tenham sido realizados e escritos a partir de metodologia rigorosamente científica relatando, portanto, resultados provavelmente verdadeiros.

Um artigo médico chega a nossas mãos através de duas vias principais 3 . Ou nos deparamos com ele ao lermos os índices das revistas - que subscrevemos ou que encontramos na biblioteca - ou, então, nós o obtemos ativamente como resultado de nossa pesquisa bibliográfica a fím de resolvermos determinado problema. De qualquer maneira, ele deve ser submetido à questão fundamental: ser lido ou ser desprezado.

O primeiro ponto para resolver esta questão é que devemos dar prioridade aos artigos originais, ou seja, aos relatos, em primeira mão, das investigações planejadas 9 . Isto não quer dizer que os editoriais, as revistas e os relatos de casos não tenham importância. Os editoriais são, em geral, comentários sobre um artigo original, que podem ampliar nossa percepção do alcance daqueles resultados, embora sempre sob o ponto de vista do editor e, não, à luz de nossa própria experiência. As revisões têm maior utilidade para a abordagem genérica e atualizada de um tema fora de nosso domínio específico. Por sua vez, os relatos de casos nos chamam a atenção para uma apresentação incomum de uma doença, ou para a própria ocorrência de uma doença rara. No entanto, os artigos que mais poderão influir em nossa prática diária são os trabalhos originais.

Algumas vantagens e dificuldades da leitura de artigos originais estão relacionados na Tabela 1. A primeira vantagem é de ordem temporal. Os artigos originais relatam os resultados, pela primeira vez, de novos experimentos ou relatam novos achados. Passará um tempo considerável até que estes novos conhecimentos apareçam em revisões, ou sejam publicados em monografias e livros-textos. Além disto, um artigo original publicado em boa revista, passa sempre pelo crivo editorial, quando reconhecidos especialistas na área analisam o trabalho, podendo recomendar modificações no texto ou mesmo sua devolução.

Outra vantagem é que os artigos originais contêm descrição detalhada dos métodos empregados, desde os critérios de seleção de pacientes aos meios de diagnósticos e às formas terapêuticas. Isto permite ao leitor verificar se os resultados são válidos e aplicáveis à sua clínica específica. Além do mais, os artigos originais contêm um sumário, em geral, de 100 a 250 palavras, que fornece objetivamente o propósito de trabalho, o modus operandi e suas conclusões. $\mathrm{O}$ texto é curto e objetivo, escrito em linguagem direta e clara.

Há, no entanto, algumas dificuldades na leitura dos artigos originais 9 . A primeira delas é seu grande número. A maioria vem publicada em periódicos da especialidade mas muitos aparecem diluidos em outras revistas, tornando o trabalho de rastreamento das revistas bastante penoso. Outra caracteristica de um artigo original 
Tabela 1. Vantagens $e$ dificuldades da leitura de artigos originais (modificado de Haynes et al. (9) ).

Vantagens

Originalidade: primeiro relato sobre novos achados

Revisão por corpo editorial

Documentação da validade e da aplicação

Sumário conciso e linguagem precisa

Dificuldades

Volume: grande número de trabalhos

Trabalhos de perspectiva estreita

Falta de avaliação explícita da validade e aplicabilidade

Intervalo entre o conhecimento e sua aplicação prática

Acesso a todos periódicos relevantes

Custo

é sua especificidade ou estreiteza de perspectiva, uma vez que o progresso da medicina se dá pelo estudo de pequenas questões relacionadas à doença e não pela descoberta súbita de uma solução genérica 9. Portanto, a maioria dos artigos trata de um pequeno aspecto do problema, enquanto a solução do problema como um todo permanece fora de seu alcance. Uma terceira dificuldade consiste na necessidade de formular o leitor um conjunto de critérios para avaliar a validade $e$ a aplicabilidade daquele artigo tanto de maneira geral quanto em relação à sua prática específica. Isto demanda uma atitude ativa que consome tempo e energia. Outro ponto que possa parecer desvantagem na leitura de artigos originais é o intervalo entre a obtenção de informações e a oportunidade de seu uso clínico. Ou seja, na maioria das vezes que o médico lê trabalhos originais ele não se defronta, naquele momento, com uma situação clínica em que a informação lhe seria imediatamente útil. No entanto, ele poderá fazer uso dela assim que aquela situação clínica ocorrer em sua prática, desde que desenvolva sistema de armazenamento e fácil levantamento desses artigos, para tê-los à mão no momento da necessidade. Por fim, outras dificuldades para a leitura de artigos originais se referem ao acesso ao grande número de peródicos em que são publicados $\mathrm{e}$ ao custo financeiro destas revistas. Estas dificuldades são minimizadas pela existência, em alguns centros, de boas bibliotecas médicas que subscrevem a maioria das revistas de maior expressão e dispõem de recursos para obtenção de cópias de trabalhos através de outras bibliotecas nacionais ou do exterior. Em cidades onde não há uma boa biblioteca médica a formação de um clube de revistas pelos médicos locais, com subscrição dos principais periódicos e sua circulação entre membros do clube, pode solucionar parcialmente o problema a um custo suportável.

\section{A escolha das revistas}

O processo de extração dos trabalhos originais, que sejam relevantes à nossa prática e que tenham sido realizados com suficiente rigor científico, é tarefa que se inicia pela seleção dos periódicos médicos que se vai manusear. A biblioteca Nacional de Medicina dos Estados Unidos indexa cerca de 250000 novos artigos por ano publicados em 3200 das 20000 revistas biomédicas 16 . O crescimento da literatura é de $6 \%$ a $7 \%$ ao ano, portanto ela duplica a cada 10 anos 12 . Isto significa que o número de periódicos também cresce nesta proporção. Daí a necessidade do desenvolvimento de estratégia para a seleção das revistas, pontencialmente de maior relevância e utilidade para a nossa prática, de acordo com o tempo disponivel. $\mathrm{Na}$ Tabela 2 são enumeradas algumas sugestões para esta estratégia.

1. Desprezar as revistas gratuitas - As revistas enviadas gratuitamente aos médicos têm, em geral, objetivos primariamente comerciais, de modo que as informações nelas contidas são suspeitas. Além disto, os artigos não são revisados por corpo editorial de especialistas na área. De maneira geral, os autores de uma investigação original, planejada e executada sob estreitos critérios científicos, não relatam seus achados em periódicos deste tipo. Portanto estas revistas não devem ocupar 0 escasso tempo do médico, que procura na literatura os meios confiáveis para a atualização de conceitos e condutas. 
Tubela 2. Sugestões para abordagem às revistas médicas.

1. Despreze as publicações enviadas gratuitamente

2. Escolha as revistas da especialidade relevantes a prática

3. Despreze as revistas gerais de pequena circulação

4 Consulte as revistas gerais de maior influência

5. Leia uma revista nacional da especialidade

6. Forme com os colegas um clube de leitura para complementar subscrições

7. Reveja regularmente os Current Contents

8. Crie seu próprio banco de referências

9. Desenvolva o hábito de freqüentar regularmente a biblioteca

2. As revistas da especialidade - O neurologista necessita do contato regular com as revistas da especialidade. Embora a ordem de preferência possa parecer questão de simpatia pessoal, ele deve usar critérios objetivos no estabelecimento das prioridades. As revistas serão mais interessantes à medida em que contiverem: (1) maior número de trabalhos originais relevantes à sua prática; (2) maior percentual de trabalhos relevantes à prática apresentando validade metodológica $e$ aplirabilidade 10,11. A partir destes dois princípios ele deve construir sua lista de preferência. Recentemente, critérios objetivos de avaliação da influência das revistas sobre a comunidade médica têm sido desenvolvidos 4,5,7. Esta influência é medida pelo chamado fator impacto, definido pelo número de citações (em todas revistas indexadas pelo Science Citation Index), num determinado ano, dos artigos publicados em determinada revista nos últimos dois anos, dividido pelo número de artigos publicados naquela revista nesse período 7,10. A frequência com que a comunidade medica mundial cita um periódico é um índice da influência ou impacto que este periódico exerce sobre ela. O fator impacto é, portanto, um ponto de referência que pode ser usado na seleção das revistas. No caso dos periódicos da especialidade, o neurologista pode escolher aquelas que julgar mais úteis para sua prática, observando os critérios acima e de acordo com seus intereses específicos e tempo disponível. $\mathrm{Na}$ Tabela 3 são enumeradas algumas das principais revistas de neurologia.

Tabela 3. Principais revistas médicas gerais em relação ao fator impacto (modificado de Garfield(7) e de Haynes et al.(9)).

The New England Journal of Medicine

The Lancet

British Medical Journal

Journal of the American Medical Association

Annals of Internal Medicine

American Journal of Medicine

Archives of Internal Medicine

Proceedings of the Society for Experimental Biology and Medicine

Acta Medica Scandinavica

Deutsche Medizinische Wochenschrift

Medical Journal of Australia

Canadian Medical Association Journal

Medicine

American Journal of the Medical Sciences

Nouvelle Presse Medicale

3. Desprezar as revistas gerais de pequena circulação - Embora pequena circulação não seja sinônimo de baixa qualidade 10, as revistas de pequena circulação, em geral, são publicações regionais, de instituições ou de sociedades médicas, que contem matéria de interesse regional, relatos de casos e revisões de literatura. As 
revistas de pequena circulação não são atrativas a pesquisadores que, naturalmente, desejam que seus estudos alcancem a maior audiência possivel.

4. Consultar as revistas gerais de maior influência - As revistas gerais de maior impacto ou influência representam fonte rica em artigos originais de grande importância nas várias especialidades. De fato, a maioria dos trabalhos mais significativos, em qualquer ramo da medicina, não é publicada em jornais médicos da especialidade ou subespecialidade, mas em revistas gerais de grande impacto 10 . Dai a necessidade do neurologista consultar sistemática e regularmente estas revistas, procurando os trabalhos reterentes a temas neurológicos. Estas revistas primam pelo rigor de sua política editorial, selecionando trabalhos de alta qualidade, que representam avanços significativos no conhecimento. $\mathrm{Na}$ Tabela 4 são relacionadas algumas das revistas de maior impacto.

Tabela 4. Principais revistas de neurologia.

Revistas Genéricas

Acta Neurologica Scandinavica

Annals of Neurology

Archives of Neurology

Brain

Canadian Journal of Neurological Sciences

Journal of Neurological Sciences

Journal of Neurology, Neurosurgery and Psychiatry

Neurology

Revue Neurologique

Revistas de Subespecialidades

Cephalalgia

Headache

Developmental Medicine and Child Neurology

Electroencephalography and Clinical Neurophysiology

Epilepsia

Journal of Clinical Neuro-Ophthalmoiogy

Movement Disorders

Muscle and Nerve

Stroke

Revisões

Cerebrovascular and Brain Metabolism Reviews

Current Opinion in Neurology and Neurosurgery

Neurologic Clinics of North America

5. Ler uma revista nacional da especialidade - O neurologista brasileiro deve acompanhar o desenvolvimento da especialidade no país, assim como se interessar pelas peculiaridades regionais da neurologia e pelo conhecimento da epidemiologia das doenças do sistema nervoso em nosso meio. Estes são os motivos principais que justificam a leitura regular de uma revista nacional de neurologia. Embora as revistas nacionais não exibam ainda uma política editorial baseada em desejável rigor científico - os artigos enviados para publicação não são submeitdos a revisão por especialistas da área especifica, que possam sugerir correções e emitir julgamento por critérios explícitos, objetivos e impessoais da validade ou não daquele artigo muitos dos artigos publicados são de elevada qualidade científica e de grande relevância e aplicabilidade à nossa prática diária.

6. Formar um clube de revistas - Quando o neurologista não dispuser em sua cidade de biblioteca médica suficientemente rica para the proporcionar a leitura das revistas de seu interesse, ele poderá formar com os colegas um clube de subscrição dos periódicos complementares. Esta medida possibilitará a todos o acesso a maior número de revistas e incentivará discussões dos artigos nelas publicados.

7. Rever regularmente os Current Contents - Os Current Contents são publicações semanais que contém os indices dos últimos números das revistas médicas. 
Seu manuseio regular propicia o conhecimento dos titulos dos trabalhos, facilitando a triagem dos artigos que serào analisados quanto a sua qualidade metodológica, a sua validade e aplicabilidade à prática. O hábito de usar regularmente os Current Contents reduz o tempo e o esforço consumidos na localização das várias revistas na biblioteca, cujos índices serão rastreados a procura dos artigos relevantes.

8. A criação do banco de referências - É dificil obter e guardar cópia de todos os artigos que possam ser úteis à nossa prática. Frequentemente também deparamos com artigos tangenciais a nosso interesse imediato, mas que se revelam úteis pouco tempo depois. A criação de banco de referências é a solução para estes problemas. Enquanto os Current Contents relacionam os índices dos periódicos publicados, outras fontes de referências como o Index Medicus, Exerpta Medica $\mathrm{e}$ outras da própria especialidade como Current Opinion in Neurology and Neurosurgery relacionam as referências por assuntos. Embora estes recursos tenham grande valor em pesquisa bibliográfica, a criação de um banco personalizado de referências tem vantagens próprias como a inclusão de artigos mais recentemente publicados, a seleção apenas daqueles que preenchem critérios de qualidade científica, de validade e de aplicabilidade à nossa prática diária, assim como a possibilidade de anotação junto àquela referência de observações que julgamos pertinentes no momento de nosso primeiro contato com o artigo. É importante lembrar, porém, que por maior que seja nosso banco personalizado de referências, ele será sempre limitado, não substituindo, portanto, os meios convencionais de levantamento das referências. $O$ uso de microcomputador simplificará a tarefa do banco personalizado, assim como o levantamento de todas as referências, pela obtenção de programas específicos.

9. O hábito de frequentar a biblioteca - A frequência regular à biblioteca é a melhor maneira de coletar as referências e os próprios artigos que devem ser lidos. Eın geral, duas horas por semana é o tempo suficiente para a realização deste trabalho 11. Há necessidade, no entanto, de considerável auto-disciplina para se ater ao manuseio daqueles periódicos já previamente selecionados, que melhor servirăo a nossos objetivos, assim como para desprezar os artigos relevantes à nossa prática mas cuja metodologia não seja satisfatória. Se não desenvolvermos esta autodisciplina, nosso tempo será consumido em atividade não produtiva. Para evitar que o mesmo número de uma revista seja manuseado mais de uma vez, no processo de triagem dos trabalhos de interesse, é conveniente fazer relação dos títulos das revistas que devemos consultar regularmente, anotando nesta relação os números já triados.

\section{Os artigos de revisão da literatura}

Os artigos de revisão da literatura podem ser úteis em três principais aspectos. Primeiro, eles podem oferecer visão geral de tema com o qual o leitor não esteja muito familiarizado, servindo então como fonte de informação e de atualização em áreas adjacentes a seu maior interesse e atuação. Segundo, eles servem como fonte de referências de trabalhos originais que merecem a avaliação do especialista 9 . Neste caso a revisão não é abordada como apreciação seletiva e crítica do estado atual do conhecimento mas apenas como listagem de trabalhos relevantes, alguns dos quais possam ter escapado à pesquisa do especialista ${ }^{6}$. Por fim, eles podem conter a meta-análise dos artigos originais aumentando o significado de seus resultados (veja abaixo).

Quando um artigo de revisão é selecionado por um dos motivos acima, algumas normas para sua apreciação devem ser consideradas a fim de que sejam descartadas revisões subjetivas, tendenciosas, sem baseamento científico ou ineficientes. Critérios explícitos para a avaliação das revisões têm sido formulados 14,16-18 e a análise cuidadosa de revisões publicadas frequentemente demonstra graves falhas quanto à metodologia científica para a identificação, avaliação e sintese das informações disponíveis 17 . O conhecimento e o uso destas normas (Tabela 5) são úteis para a leitura crítica das revisões da literatura, evitando dispêndio de tempo e energia além do risco das conclusões errôneas.

Primeiro, todo artigo de revisão deve ser escrito para responder uma pergunta. Esta questão deve estar clara, bem formulada e precisa no início do artigo. Depois, as fontes e os métodos de procura dos trabalhos devem ser explicitados. Como é praticamente impossível cobrir toda a literatura existente 19, o leitor precisa saber qual segmento da literatura foi pesquisado - se em um determinado tempo, se restrito a uma língua ou a um país, etc. - e que estratégia foi empregada. Atual- 
Tabela 5. Questóes para avalią̧̃̃o dos artigos de revisão.

\author{
O propósito específico da revisão foi bem definido? \\ As fontes pesquisadas foram identificadas? \\ Os métodos de levantamento da literatura foram estabelecidos? \\ Que normas determinaram a inclusão e exclusão de artigos? \\ A validade da metodologia dos artigos foi avaliada? \\ A informação foi integrada e as limitações e inconsistências avaliadas? \\ Foi realizada meta-análise dos trabalhos incluidos? \\ As conclusões das revisões são baseadas nos dados citados? \\ Foi incluído sumário dos achados pertinentes? \\ Os problemas remanescentes e não resolvidos foram relacionados? \\ Diretivas para novas pesquisas foram propostas?
}

mente, tais estratégias incluem o uso de databases bibliográficas com a especificação das palavras-chave. Outros meios, como o uso dos Current Contents, de outros artigos de revisão e de referências em artigos originais, são também frequentemente empregados.

A definição dos critérios de inclusão e exclusão de trabalhos na revisão deve ser claramente exposta, pois muitos cios artigos publicados são irrelevantes à questão em estudo ou, frequentemente, apresentam deficiências metodológicas que invalidam os resultados. As revisões de um mesmo tópico podem chegar a conclusóes até opostas, dependendo da seleção dos artigos incluídos 18. É importante, portanto, que os autores especifiquem como os artigos foram escolhidos, definindo as questões e determinando os elementos metodológicos. Assim, por exemplo, numa revisão de algum aspecto da doença de Alzheimer, os autores devem explicitar que os trabalhos sobre outras formas de demência foram excluídos, que apenas aqueles artigos contendo avaliação do estado mental por métodos comprovadamente válidos foram considerados, e como os grupos de pacientes foram formados, ou seja, se a revisão está limitada apenas aos estudos com grupos randomizados, ou se foram incluidos também artigos com grupos de coortes ou estudos retrospectivos.

As revisões devem também empregar métodos padronizados de avaliação da validade das informações originais disponiveis. Nesta avaliação estão incluidos o propósito do trabalho, o seu protocolo, a metodologia usada em relação à população investigada e à intervenção realizada, assim como os resultados obtidos. Apenas artigos originais com rigor metodológico devem ser incluídos. Os trabalhos originais devem ser analisados cuidadosamente e suas falhas e inconsistências, expostas. Por sua vez, as informações válidas poderão ser integradas através da meta-análise 8,15 A meta-análise é a disciplina que revê e combina estatisticamente as informações de pesquisas originais 21 . Os propósitos da meta-análise são: (1) aumentar o poder estatístico dos resultados finais e dos subgrupos; (2) resolver incertezas quando há discordâncias entre os resultados individuais dos trabalhos; (3) melhorar as estimativas; e (4) responder a questões não formuladas nos artigos originais 21 . Os métodos da meta-análise incluem a apreciação crítica de cada estudo desde sua finalidade, modo de realização e suas conclusões. Os resultados são então combinados de acordu com os dados em relação às características dos pacientes, seus diagnósticos e as terapias envolvidas. Cada um destes pontos é analisado, por sua vez, em relação a suas semelhanças, identidade dos critérios de avaliação e das condutas tomadas, a fim de que a combinação das casuísticas seja processada. Métodos de análise estatística para comparação e combinação de resultados são imprescindíveis 2,21 . A identificação das variabilidades das observações, dos preconceitos e falhas é fundamental.

Nas revisões as conclusões são justificáveis apenas quando todo o processo descrito, da coleta à análise e, daí, à integração dos dados dos trabalhos originais, é realizado sistemática e completamente. As revisões em que os resultados não são estatisticamente combinados devem expor claramente as bases para as conclusões, assim como explicar os resultados conflitantes 18. Quando a informação é integrada um sumário dos achados pertinentes é útil.

Por fim, as revisões são importantes na identificação das lacunas ainda existentes no conhecimento daquela questão e na proposição de novas investigações para preenchimento dessas falhas. Uma boa revisão, portanto, aborda não apenas o estado atual do conhecimento mas, também, do não-conhecimento 13. 


\section{REFERENCIAS}

1. Bernier CL, Yerkey AN. Cogent Communication: Overcoming Information Overload. Westport: Greenwood Press, 1979, p 39.

2. Cooper HM, Rosenthal R. Statistical versus traditional procedures for summarizing research findings. Psychol Bull 1980, 87:442-449.

3. Department of Clinical Epidemiology and Biostatistics, McMaster University Health Sciences Centre. How to read clinical journals: $I$. Why to rcad them and how to start read them critically. Can Med Assoc J 1981, 124:555-558.

4. Garfield E. Citation indexing for studying science. Nature 1970, 227:669-671.

5. Garfield E. Citation analysis as a too' in journal evaluation. Nature 1971, 178:471-479.

6. Garfield E. The 1983 articles most cited in 1983 and 1984: 1. Life sciences. Curr Contents 1985, 28:3-18.

7. Garfield $\mathbf{E}$. Which journals have the greatest impact? Ann Intern Med 1986, 105:313-320.

8. Glass GV. Primary, secondary and meta-analysis of research. Educ Res 1976, 5:3-8.

9. Haynes RB, McKibbon KA, Fitzgerald D, Guyatt G, Walker CJ, Sacktt DL. How to keep up with the medical literature: I. Why try to keep up and how to get started. Ann Intern Med 1986, 105:149-153.

10. Haynes RB, Mckibbon KA, Fitzgerald D, Guyatt G, Walker CJ, Sackett DL. How to keep up with the medical literature: II. Deciding which journals to read regularly. Ann Intern Med 1986, 105:309-312.

11. Haynes RB, McKibbon KA, Fitzgerald D, Guyatt G, Walker CJ, Sackett DL. How to keep up with the medical literature: III. Expanding the number of journals you read regularly. Ann Intern Med 1986, 105:474-478.

12. Houghton B. Scientific Periodicals: Their Historical Development, Characteristics and Control. Hamden: Linnet, 1974, p 106.

13. Huth EJ. How to Write and Publish Papers in the Medical Sciences. Philadelphia ISI Press, 1982, p 64.

14. Huth EJ. Needed: review articles with more scientific rigor. Ann Intern Med 1987, $106: 470-471$.

15. Light RJ, Smith PV. Accumulating evidence: proceoures for resolving contradictions among different research studies. Harvard Educ Rev 1971, 41:429-471.

16. Morgan PP. Review articles: 2. The literature jungle. Can Med Assoc J 1986, 134:98-99.

17. Mulrow $C D$. The medical review article: state of the science. Ann Intern Med 1987, $106: 485-488$.

18. Oxman AD, Guyatt GH. Guidelines for reading literature reviews. Can Med Assoc J 1988, 138:697-703.

19. Poynard T, Conn HD. The retrieval of randomized clinical trials in liver disease from the medical literature. Controlled Clin Trials 1985, 6:271-279.

2C. Roland CG, Kirkpatrick RA. Time lapse between hypothesis and publication in medical sciences. N Engl $J$ Med 1975, 292:1273-1276.

21. Sacks HS, Berrier J, Reitman D, Ancona-Birk, VA, Chalmers TC. Meta-analysis of randomized controlled trials. N Engl J Med 1987, 316:450-455.

22. Warren KS (ed). Coping with the Biomedical Literature: a Primer for the Scientist and the Clinician. New York: Praeger, 1981 